\title{
Dietary supplementation with hydroxypropyl-distarch phosphate from waxy maize starch increases resting energy expenditure by lowering the postprandial glucose-dependent insulinotropic polypeptide response in human subjects
}

\author{
Akira Shimotoyodome ${ }^{1 *}$, Junko Suzuki ${ }^{1}$, Yoji Kameo ${ }^{2}$ and Tadashi Hase ${ }^{1}$ \\ ${ }^{1}$ Biological Science Laboratories, Kao Corporation, 2606 Akabane, Ichikai-machi, Haga-gun, Tochigi 321-3497, Japan \\ ${ }^{2}$ Health Care Food Research Laboratories, Kao Corporation, 2-1-3 Bunka, Sumida-ku, Tokyo 131-8501, Japan \\ (Received 6 September 2010 - Revised 25 November 2010 - Accepted 14 December 2010 - First published online 22 February 2011)
}

\begin{abstract}
The aim of the present study was to investigate the effects of hydroxypropyl-distarch phosphate (HDP) supplementation on postprandial energy metabolism and glucose-dependent insulinotropic polypeptide (GIP) in human subjects. A total of ten healthy male subjects, with a mean BMI of 23.6 (SEM 1.3 ) kg/m², age 35.2 (SEM 1.9) years and body weight 71.1 (SEM 4.0 ) kg, participated in a randomised, cross-over, intervention study with two different test meals $(1673.6 \mathrm{~kJ})$ containing either waxy maize starch or HDP from waxy maize starch (degree of substitution $0 \cdot 154$, P content $0 \cdot 004 \%$ ). Resting energy expenditure (REE) and blood concentrations of various biomarkers were measured at fasting and up to $180 \mathrm{~min}$ postprandially. Indirect calorimetry showed that the HDP meal caused higher REE $(P<0.05)$ and fat utilisation $(P<0.001)$ than the waxy maize starch meal. The HDP meal led to significantly lower postprandial glucose $(P<0.05)$, insulin $(P<0.05)$ and GIP $(P<0.05)$ responses than the waxy maize starch meal. Both postprandial REE $(R-0.576, P<0.01)$ and fat utilisation $(R-0.514, P<0.05)$ were negatively correlated with the postprandial GIP response, but not with the glucose and insulin responses. In conclusion, dietary supplementation with HDP lowers postprandial GIP and increases postprandial REE and fat utilisation in healthy humans. An HDP-rich diet may therefore have beneficial implications in weight management. Further studies are required to confirm the efficacy in overweight or obese subjects, and to determine the precise mechanisms.
\end{abstract}

Key words: Fat utilisation: Glucose-dependent insulinotropic polypeptide: Resting energy expenditure

Recently, we have observed that dietary supplementation with a particular type of resistant starch (RS), namely hydroxypropyldistarch phosphate from waxy maize starch (HDP, RS4 type $\mathrm{e}^{(1)}$ ), increases fat catabolism compared with the equivalent amount of high-amylose maize starch (RS2 type ${ }^{(1)}$ ), and thereby attenuates high-fat diet-induced adipogenesis in mice $^{(2)}$. Chemically modified starch including HDP is more resistant to retrogradation, has a higher viscosity, and is more stable in response to acids and a high temperature than native starch. Therefore, chemically modified starch is commonly used by the food industry to improve the physical properties of various food items, including baby food. Many investigators have shown that chemically modified starch shows a reduced susceptibility to enzymes in vitro and in vivo compared with the corresponding unmodified $\operatorname{starch}^{(3,4-7)}$ as well as other types of $\mathrm{RS}^{(1,8-10)}$.
Previous studies have shown a decrease in postprandial glycaemic or insulinaemic responses to RS (mostly RS2-type) ingestion relative to digestible starch consumption ${ }^{(11,12-15)}$, whereas some have reported no change $\mathrm{e}^{(16-18)}$. Since RS contains a reduced amount of available energy, a diet rich in RS may be useful in the treatment of obesity. However, previous studies have shown that postprandial energy expenditure did not increase, but rather decreased after the intake of RS compared with that for digestible starch, since RS is not absorbed as glucose in the small intestine of healthy humans. A study by Ranganathan et $a l .{ }^{(18)}$ has shown that amylose-rich starch (RS2) has no thermogenic effect either alone or mixed with glucose in humans. In addition, Tagliabue et al. ${ }^{(19)}$ concluded that raw (ungelatinised) starch (RS2) has no thermogenic effect during the $5 \mathrm{~h}$ after its intake, and that it does not influence the size of the thermic response to digestible starch. Thus, it is still unclear whether RS consumption is beneficial

Abbreviations: AUC, area under the curve; GIP, glucose-dependent insulinotropic polypeptide; HDP, hydroxypropyl-distarch phosphate; REE, resting energy expenditure; RS, resistant starch.

*Corresponding author: A. Shimotoyodome, email shimotoyodome.akira@kao.co.jp 
to control energy homeostasis and improve obesity in humans.

Our previous study has shown that postprandial blood glucose and insulin levels are decreased after the consumption of HDP as well as high-amylose maize starch compared with those for the corresponding digestible starch (waxy maize starch) ${ }^{(2)}$. Very interestingly, the postprandial blood response of a gut-derived hormone, glucose-dependent insulinotropic polypeptide (GIP), was significantly lowered by HDP, but not by high-amylose maize starch consumption compared with that for waxy maize $\operatorname{starch}^{(2)}$. Recently, GIP has been suggested to be a key regulator of adipocyte biology and energy metabolism, stimulating efficient fat deposition in body stores ${ }^{(20,21)}$. The inhibition of GIP signalling enhanced fat oxidation and energy expenditure, and reduced high-fat diet-induced obesity in mice ${ }^{(22,23)}$. Daousi et al. ${ }^{(24)}$ showed that a higher blood GIP level reduces resting energy expenditure (REE) in healthy humans. Thus, the inhibition of dietinduced obesity and increased fat catabolism in mice after the consumption of HDP seems to be attributable to a lower postprandial GIP response. While there is currently no evidence regarding the relationship between HDP ingestion and either postprandial energy consumption or the GIP response in humans, dietary supplementation with HDP would be expected to exert beneficial effects to improve obesity. It is important that the above-mentioned parameters be carefully defined before designing and conducting longterm, prospective HDP-feeding studies in human subjects.

The primary aim of the present study was to clarify the effect of dietary HDP supplementation on postprandial energy metabolism and the blood GIP response in human subjects. We examined how the single ingestion of a meal supplemented with either waxy maize starch or HDP affects postprandial energy metabolism and blood metabolites and hormones in healthy human subjects.

\section{Materials and methods}

\section{Materials}

Waxy maize starch and HDP from waxy maize starch (degree of substitution $0 \cdot 154, \mathrm{P}$ content $0 \cdot 004 \%$, swelling capacity $15.0 \mathrm{ml} / \mathrm{g}$, water holding capacity $17.3 \mathrm{~g} / \mathrm{g}$, paste viscosity $1.65 \mathrm{Pas}\left(6.25 \%(\mathrm{w} / \mathrm{w}), 27^{\circ} \mathrm{C}\right)$, Brabender viscosity $380 \mathrm{BU}$ $\left(5 \%(\mathrm{w} / \mathrm{w}), 93^{\circ} \mathrm{C}\right)$, onset, peak and conclusion temperatures of gelatinisation 47,64 and $78^{\circ} \mathrm{C}$, respectively) were obtained from National Starch Food Innovation (Tokyo, Japan).

\section{Subjects}

A total of ten male volunteers (age $35 \cdot 2$ (sem 1.9) years, body weight 71.1 (SEm 4.0$) \mathrm{kg}$, BMI $23.6($ SEm 1.3$) \mathrm{kg} / \mathrm{m}^{2}$ ) showed no abnormalities on physical examination. None of the subjects had a family history of diabetes. For $2 \mathrm{~d}$ before the study, all subjects abstained from alcohol and exercise. The present study was conducted according to the guidelines laid down in the Declaration of Helsinki, and all procedures involving human subjects were approved by the Ethical Committee of
Kao Corporation. Written informed consent was obtained from all subjects. All subjects were paid for their participation in the experiment.

\section{Pancake test meal}

A pancake batter was prepared using $18.6 \%$ pregelatinised test starch, $3.7 \%$ sugar, $7 \cdot 5 \%$ maltodextrin (Fujioligo $\# 450^{\circledR}$; Nihon Shokuhin Kako Company, Limited, Tokyo, Japan), 19.6\% egg, 9\% defatted milk powder and 37.3\% water. Pancakes were prepared in the Food Manufacturing Facility of Kao Tokyo Institute following the institutional sanitary guidelines. The meal was consumed together with water $(180 \mathrm{ml})$. The nutritional composition of the test meal is shown in Table 1.

\section{Protocol}

All subjects had the same dinner containing 25.4 g of fat (total energy content $3740 \mathrm{~kJ}$ ( $894 \mathrm{kcal}$ )) between 20.00 and 20.30 hours, and refrained from food intake and drinking-water for at least 12 and $3 \mathrm{~h}$, respectively, before admission to the institute, between 08.00 and 08.30 hours on the study day.

At 09.00 hours, before meal ingestion, baseline blood samples (approximately $200 \mu \mathrm{l}$ ) were collected for fasting glucose, insulin, GIP and TAG by pricking a finger with a singleuse sterile capillary blood-sampling device (BD Genie lancet; Becton-Dickinson, Tokyo, Japan) into a heparinised capillary tube (75 mm length; Drummond Scientific Company, Broomall, PA, USA). The blood samples were transferred into capillary blood collection tubes (CAPIJECT with EDTA-2Na; Terumo Medical Company, Tokyo, Japan) containing a dipeptidyl peptidase IV inhibitor (Millipore, Tokyo, Japan) and maintained on ice until plasma preparation. Subjects ingested a test meal for 5-12 min thereafter. All subjects were studied on two occasions separated by at least $7 \mathrm{~d}$ in a randomised,

Table 1. Nutritional composition of the test meals

\begin{tabular}{|c|c|c|}
\hline Meals & $\begin{array}{l}\text { Waxy maize } \\
\text { starch meal }\end{array}$ & HDP meal \\
\hline Energy (kJ) & $1673 \cdot 6$ & $1673 \cdot 6$ \\
\hline Waxy maize starch $(\mathrm{g})$ & $38 \cdot 0$ & 0 \\
\hline HDP from waxy maize starch $(\mathrm{g})$ & 0 & $38 \cdot 0$ \\
\hline Sucrose $(g)$ & $7 \cdot 5$ & $7 \cdot 5$ \\
\hline Maltodextrin (g) & $15 \cdot 3$ & $15 \cdot 3$ \\
\hline Total wet weight (g) & $204 \cdot 0$ & $204 \cdot 0$ \\
\hline Total carbohydrate $(\mathrm{g})$ & $62 \cdot 6$ & $62 \cdot 6$ \\
\hline Total fat (g) & $10 \cdot 8$ & $10 \cdot 8$ \\
\hline Total protein $(\mathrm{g})$ & $11 \cdot 2$ & $11 \cdot 2$ \\
\hline Total fibre (g) & 0 & 0 \\
\hline Degree of gelatinisation (\%)* & $67 \cdot 5$ & $85 \cdot 7$ \\
\hline Glycaemic index $†$ & 100 & $66 \cdot 7$ \\
\hline Glycaemic load $\ddagger$ & $62 \cdot 6$ & $41 \cdot 4$ \\
\hline
\end{tabular}

HDP, hydroxypropyl-distarch phosphate; AUC, area under the curve.

* The degrees of gelatinisation were determined by the $\beta$-amylase-pullulanase method $^{(33)}$.

$\dagger$ Relative glycaemic index was calculated as the mean $\mathrm{AUC}_{\text {glucose }} 120 \mathrm{~min}$ of each

subject $(n 10)$ relative to that of the waxy maize starch meal as a control (100).

$\ddagger$ Glycaemic load = glycaemic index $/ 100 \times$ total carbohydrate $(\mathrm{g})$. 
single-blinded order (waxy maize starch or HDP). Subjects remained in a sedentary position throughout the study period. Over the postprandial period, blood samples were taken at 30, 60, 90, 120 and $180 \mathrm{~min}$. After centrifugation, plasma was stored at $-80^{\circ} \mathrm{C}$ until analysis.

\section{Indirect calorimetry}

Subjects were adapted to a room maintained at $23 \pm 1^{\circ} \mathrm{C}$, with a relative humidity of $40 \pm 10 \%$ for $15 \mathrm{~min}$. Respiratory metabolic performance in the sedentary condition was measured using an open-circuit ventilated hood system (VO2000 ${ }^{(25)}$; Medical Graphics Company, St Paul, MN, USA) six times (before the meal $(-15$ to $0 \mathrm{~min})$ and at $30-45,60-75$, 90-105, 120-135, 150-165 and 180-195 min after the meal). Gas exchange rates were recorded on every third expiration. For baseline data, an equilibrium period of $5 \mathrm{~min}$ was allowed; data from the last $10 \mathrm{~min}$ were averaged for the calculation of $\operatorname{REE}(\mathrm{kJ} / \mathrm{d})$ and the RER.

The measured values of $\mathrm{VO}_{2}$ and $\mathrm{CO}_{2}$ production $\left(\mathrm{VCO}_{2}\right)$ were converted to the REE using the Weir equation ${ }^{(26)}$ with a specific software program (m-Graph ${ }^{\circledR}$; S\&ME, Inc., Tokyo, Japan). The RER ( $\left.\mathrm{RER}=\mathrm{VCO}_{2} / \mathrm{VO}_{2}\right)$ and substrate utilisation were also estimated from the gas exchange data on the software. RMR $(\mathrm{kJ} / \mathrm{d})$ was calculated as the average REE of the test period.

\section{Plasma contents and hormone levels}

Blood glucose was determined by employing a blood glucose self-monitoring device (ACCU-CHECK COMFORT ${ }^{\circledR}$; Roche Diagnostics Company, Tokyo, Japan) immediately after blood collection. Plasma TAG and NEFA levels were determined using spectrophotometric methods (Triglyceride E-test and NEFA-C test assay kits, respectively; Wako Pure Chemical Industries, Limited, Osaka, Japan). Plasma insulin was determined using a Human Insulin ELISA kit (Morinaga Institute of Biological Science, Inc., Yokohama, Japan). Total GIP was measured employing an ELISA kit (Linco Research, Inc., St Charles, MI, USA).

\section{Statistical analysis}

Numerical data are expressed as means with their standard errors. Glycaemic, insulinaemic and GIP responses were assessed by calculating the incremental area under the curve (AUC) using the trapezoid rule. Student's $t$ tests after a preliminary $F$ test of the homogeneity of within-group variance or paired $t$ tests were used when comparing values between the groups. Changes over time and between the groups were compared using two-way repeated-measures ANOVA, and Pearson's correlation coefficients were obtained to estimate the linear correlation between two parameters (STATVIEW for Windows version 5.0; SAS Institute, Inc., Cary, NC, USA). Differences were considered significant when the error probability was smaller than $0 \cdot 05$.

\section{Results}

Postprandial metabolic response to test meal ingestion in healthy human subjects

We examined the effect of HDP supplementation of the diet on energy metabolism in healthy humans. $\mathrm{VO}_{2}$ and $\mathrm{REE}$ tended to be higher in the HDP meal group than in the waxy maize starch meal group, and there was a significant increase in both $\mathrm{VO}_{2}$ (Fig. 1(a)) and REE (Fig. 1(c)) at 180 min after the consumption of the HDP-compared with that for the waxy maize starch meal. The average $\mathrm{AUC}_{\mathrm{REE}}$ for $180 \mathrm{~min}$ after HDP meal ingestion was higher than that after waxy maize starch meal ingestion (126.4 (sem 31.0) $v$. 73.6 (sem 37.2) kJ, respectively, $P<0.05$ ). The RER was significantly lower in the HDP meal group than in the waxy maize starch meal group (Fig. 1(b)). Fat utilisation (Figs. 1(d) and 2(a)) during the experimental period $(180 \mathrm{~min})$ was significantly higher in the HDP subjects than in the waxy maize starch-ingesting subjects. Carbohydrate utilisation was significantly lower after the ingestion of the HDP meal than after the waxy maize starch meal (Figs. 1(e) and 2(b)). Postprandial increase in the RMR tended to be higher after HDP meal ingestion $(953.5 \mathrm{~kJ} / \mathrm{d})$ than that for the waxy maize starch meal $(528 \cdot 5 \mathrm{~kJ} / \mathrm{d})$.

\section{Postprandial blood metabolite and hormone responses in healthy human subjects}

Postprandial blood glucose, insulin, and GIP and TAG responses of human subjects to the two pancake meals are shown in Fig. 3. The initial blood glucose, insulin, TAG and NEFA levels in subjects were similar between the two meals. The fasting levels of GIP were significantly different between the two meals (Table 2).

Ingestion of the test meal increased plasma glucose levels for $60 \mathrm{~min}$, and, thereafter, the levels declined, returning to baseline after $180 \mathrm{~min}$ (Fig. 3(a)). The insulin level also peaked at $60 \mathrm{~min}$ after test meal ingestion and reduced thereafter, returning to baseline after $180 \mathrm{~min}$ (Fig. 3(b)). Blood GIP levels peaked at $120 \mathrm{~min}$ after meal consumption (Fig. 3(c)). TAG levels increased steadily during the $180 \mathrm{~min}$ test period (Fig. 3(d)). The plasma concentration of NEFA declined from the initial level up to $90 \mathrm{~min}$ after meal ingestion, and remained at a lower level up to the end of the test period (data not shown).

The glucose responses tended to be lower up to $60 \mathrm{~min}$ after the subjects consumed the HDP meal than those for the waxy maize starch meal. The late blood glucose response (90-120 min) was significantly lower after the ingestion of the HDP meal than that for the waxy maize starch meal (Fig. 3(a)). The peak blood glucose concentration $\left(C_{\max }\right.$; Table 2$)$ and incremental blood glucose area (AUC glucose $_{180}$ min; Fig. 4(a)) after the ingestion of the HDP meal were significantly lower than those after waxy maize starch meal consumption.

The maximum concentration (Table 2), maximum increase (Fig. 3(b) and (c)) and postprandial response (AUC for $180 \mathrm{~min}$, Fig. 4(b) and (c)) of both insulin and GIP were significantly lower after the subjects consumed the HDP meal 

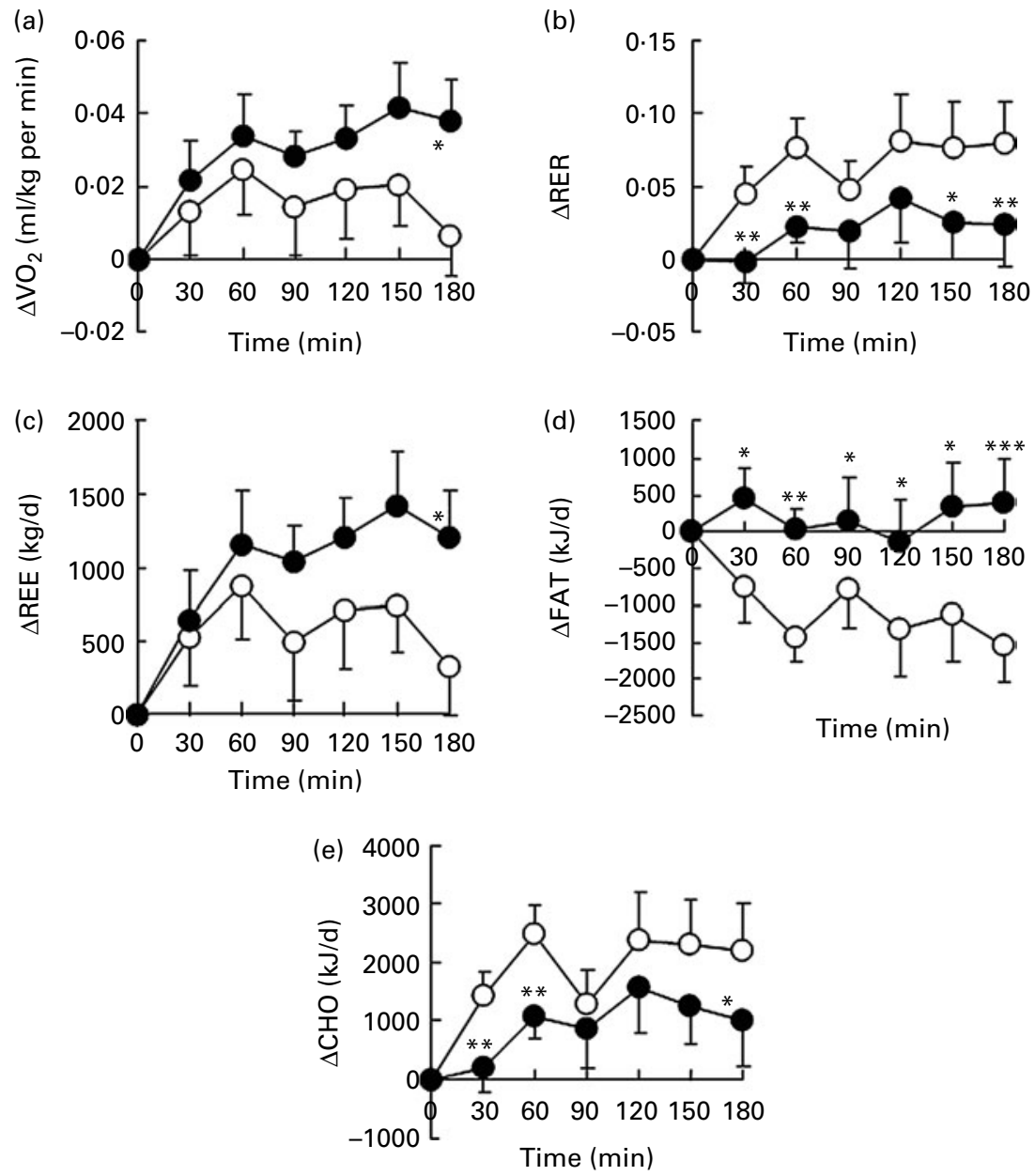

Fig. 1. (a) Increase in $\mathrm{O}_{2}$ consumption $\left(\mathrm{VO}_{2}\right)$, (b) the $\mathrm{RER}$, (c) resting energy expenditure (REE), (d) fat utilisation (FAT) and (e) carbohydrate utilisation (CHO) for up to $180 \mathrm{~min}$ after the consumption of the test meal containing waxy maize starch $(O)$ or hydroxypropyl-distarch phosphate (HDP; from waxy maize starch) ( $)$ in overnightfasted healthy male subjects. Baseline values $(0 \mathrm{~min})$ were obtained immediately before meal ingestion. Values are means, with standard errors represented by vertical bars (ten subjects per group). Statistical analysis was conducted using the paired $t$ test. Mean values were significantly different: ${ }^{\star} P<0 \cdot 05$, ${ }^{\star \star} P<0.01,{ }^{\star \star \star} P<0 \cdot 001$.

than those after the waxy maize starch meal. No significant differences were observed in the levels of TAG (Figs. 3(d) and $4(\mathrm{~d})$ ) and NEFA (Table 2). Two-way repeated-measures ANOVA revealed that the main effect of the meal was significant for postprandial increases in blood glucose, insulin and GIP, but not for that in TAG (Table 3). Meal $\times$ time interaction was not significant for any blood metabolic variables.

Correlation analysis showed that postprandial energy utilisation was significantly associated with the postprandial GIP response, but not with the blood glucose or insulin response (Table 4). Postprandial energy expenditure (Fig. 5(a) and (c)) and fat utilisation (Fig. 5(b) and (d)) were inversely correlated with either the maximum increment $\left(\Delta C_{\max }\right.$ (GIP); Fig. 5(a) and (b)) or overall response $\left(\mathrm{AUC}_{\mathrm{GIP}} 180 \mathrm{~min}\right)$ of GIP over the test period. Carbohydrate utilisation was not associated with the GIP response (Table 4).

\section{Discussion}

The first finding in the present study was that the replacement of digestible starch (waxy maize starch) with HDP in a mixed meal containing fat and protein stimulated postprandial REE, especially fat oxidation, in human subjects. The significant increase in REE in response to the HDP meal is not driven by disparate responses among subjects, as eight of the ten subjects studied showed a greater energy expenditure in response to HDP meal consumption relative to waxy maize starch meal consumption. The study also found that the meal containing HDP induced a lower postprandial GIP response than the waxy maize starch meal as well as a reduction of postprandial glycaemia and insulinaemia. These results are consistent with our previous observations in animals, which showed lower postprandial blood glucose and hormonal responses and greater fat oxidation after the consumption of HDP as compared with digestible $\operatorname{starch}^{(2)}$.

To our knowledge, this is the first study in which RS4-type RS was given to subjects as part of a mixed meal, which is balanced in terms of macronutrients, and compared with a digestible starch meal. The replacement of starch with HDP in the meal increased postprandial energy expenditure by more than $70 \%$ over the $3 \mathrm{~h}$ post-meal ingestion period, while lowering the postprandial glycaemic response by 

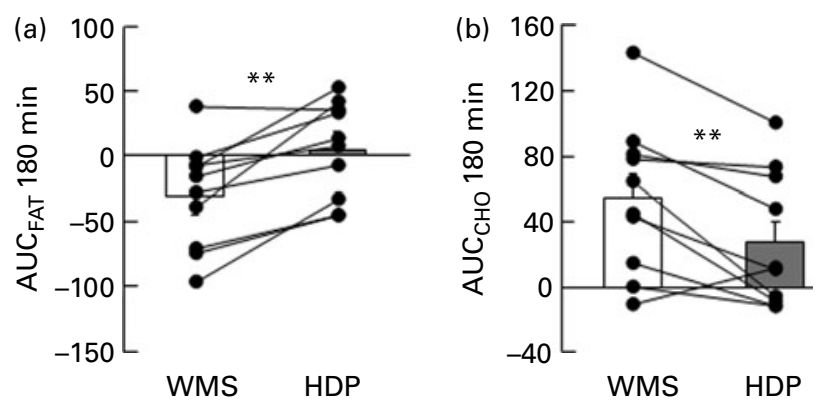

Fig. 2. Incremental area under the curve (AUC) for (a) fat and (b) carbohydrate $(\mathrm{CHO})$ utilisation up to $180 \mathrm{~min}$ after the consumption of the test meal containing waxy maize starch (WMS, $\square$ ) or hydroxypropyl-distarch phosphate (HDP; from waxy maize starch, $\square$ ) in overnight-fasted healthy male subject subjects. Baseline values $(0 \mathrm{~min})$ were obtained immediately before meal ingestion. Values are means, with standard errors represented by vertical bars (ten subjects per group). Statistical analysis was conducted using the paired $t$ test. ${ }^{\star *}$ Mean values were significantly different $(P<0.01)$.

approximately $40 \%$, relative to that with waxy maize starch. The present results seem to contradict the general perception that RS (mostly raw starch or high-amylose starch) ingestion leads to a lower postprandial energy expenditure than digestible starch. However, previous studies investigating the effect of RS on postprandial energy expenditure have a limitation in that the participants were given test meals that were composed of mainly carbohydrates as an energy substrate. Tagliabue et al. ${ }^{(19)}$ showed that postprandial energy expenditure was lower after the intake of raw potato starch (RS2) than that of pregelatinised potato starch (digestible starch), since raw potato starch is not absorbed as energy (glucose) in the small intestine. In their study, RS (raw potato starch) and the corresponding digestible starch (pregelatinised potato starch) were simply mixed into fruit syrup ${ }^{(19)}$. In another study by Ranganathan et al. $^{(18)}$, high-amylose maize starch (RS2) had no thermogenic effect either alone or mixed with glucose in human subjects. However, the test meals in previous studies did not supply dietary fat as an energy substrate. In contrast, the test diets in the present study were balanced at $30.7 \%$ carbohydrate $(62.6 \%$ as energy), $5.3 \%$ fat $(24.3 \%$ as energy) and $5.5 \%$ protein $(11 \cdot 2 \%$ as energy). The postprandial increase in energy expenditure, especially fat utilisation, after HDP meal ingestion may be due to the supply of dietary fat as an energy substrate from the test meal in the present study.

To study the underlying mechanism of increased energy expenditure and fat utilisation after the HDP meal, we examined the correlation between the metabolic variables and energy utilisation. Both energy expenditure and fat utilisation were inversely associated with the postprandial GIP response, but not with either the glycaemic or insulin response. The lower the amount of GIP secreted into the circulation from the small intestine in response to the mixed meal, the more fat was oxidised systemically as an energy substrate.

The accumulated evidence shows that GIP may be a key regulator of energy metabolism and fat deposition in body stores $^{(20,21)}$. The inhibition of either GIP signalling ${ }^{(23)}$ or GIP
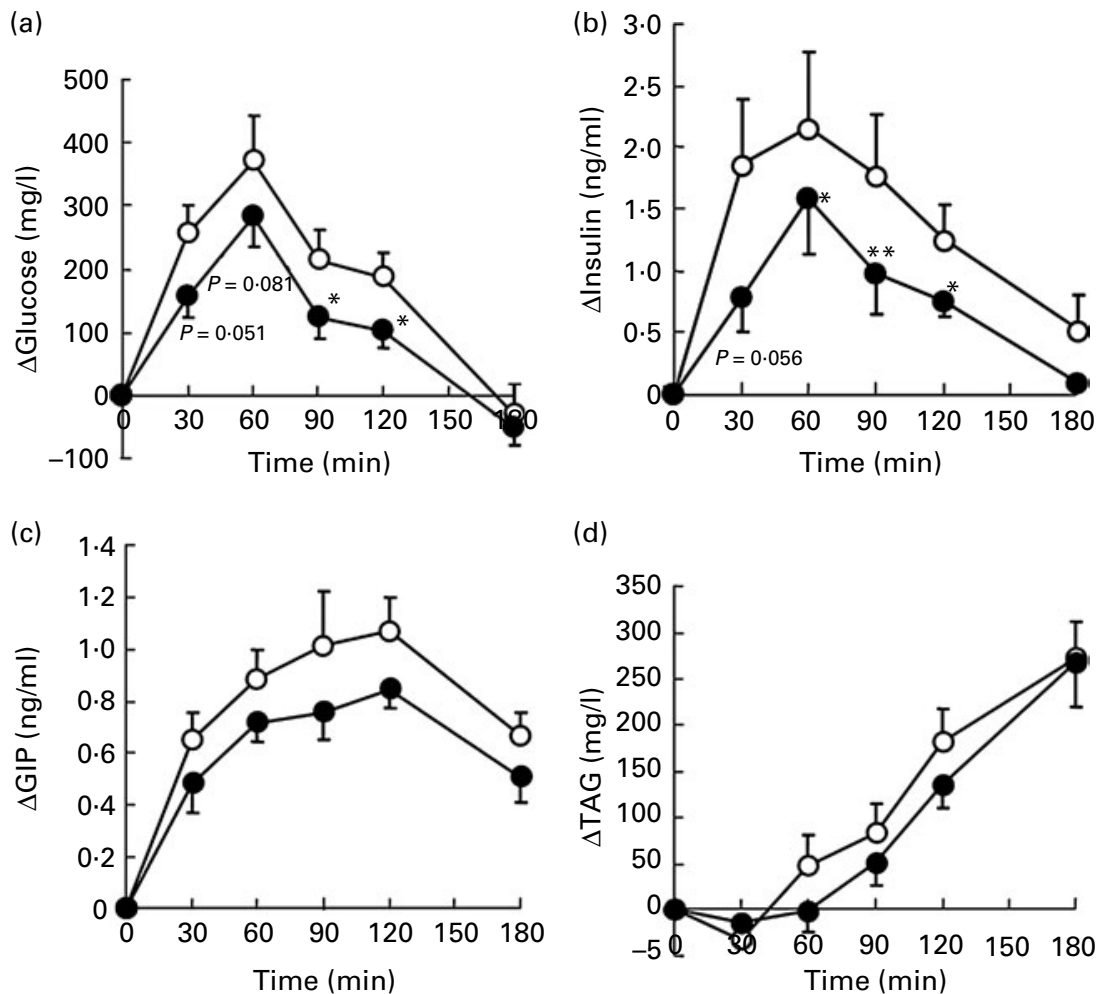

(d)

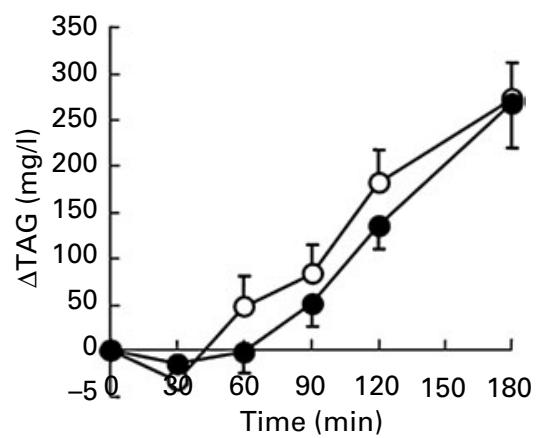

Fig. 3. Increase in (a) blood glucose, (b) insulin, (c) glucose-dependent insulinotropic polypeptide (GIP) and (d) TAG concentrations for up to 180 min after the consumption of the test meal containing waxy maize starch $(O)$ or hydroxypropyl-distarch phosphate (HDP; from waxy maize starch, $\bullet$ ) in overnight-fasted healthy male subjects. Baseline values $(0 \mathrm{~min})$ were obtained immediately before meal ingestion. Values are means, with standard errors represented by vertical bars (ten subjects per group). Statistical analysis was conducted using the paired $t$ test. Mean values were significantly different: ${ }^{*} P<0.05,{ }^{* *} P<0 \cdot 01$. 
Table 2. Initial $\left(C_{0}\right)$ and maximum $\left(C_{\max }\right)$ concentrations of blood metabolic variables before and after ingestion of the waxy maize starch (WMS) or hydroxypropyl-distarch phosphate (HDP) meal*

(Mean values with their standard errors; ten subjects per group)

\begin{tabular}{|c|c|c|c|c|c|c|c|c|c|c|}
\hline \multirow[b]{3}{*}{ Variables } & \multicolumn{5}{|c|}{$C_{0}$} & \multicolumn{5}{|c|}{$C_{\max }$} \\
\hline & \multicolumn{2}{|c|}{ WMS } & \multicolumn{2}{|c|}{ HDP } & \multirow[b]{2}{*}{$P$} & \multicolumn{2}{|c|}{ WMS } & \multicolumn{2}{|c|}{ HDP } & \multirow[b]{2}{*}{$P$} \\
\hline & Mean & SEM & Mean & SEM & & Mean & SEM & Mean & SEM & \\
\hline Glucose (mg/l) & 935 & 22 & 961 & 29 & NSt & 1392 & 41 & 1282 & 33 & $<0.001$ \\
\hline Insulin (ng/ml) & 0.39 & 0.06 & 0.38 & 0.03 & NSt & $3 \cdot 1$ & 0.7 & $2 \cdot 1$ & 0.5 & $<0.05$ \\
\hline GIP (ng/ml) & $2 \cdot 1$ & $0 \cdot 1$ & 1.9 & 0.1 & $<0.01$ & $3 \cdot 3$ & 0.2 & $2 \cdot 9$ & 0.2 & $<0.01$ \\
\hline TAG (mg/l) & 921 & 12 & 877 & 96 & NSt & 1197 & 140 & 1147 & 121 & NSt \\
\hline NEFA (mEq/l) & 0.18 & 0.02 & 0.18 & 0.02 & NS† & $0.05 \ddagger$ & 0.01 & $0.05 \ddagger$ & 0.01 & NSt \\
\hline
\end{tabular}

GIP, glucose-dependent insulinotropic polypeptide.

* Statistical analysis was conducted using the paired $t$ test.

$\dagger$ Values were not significantly different $(P>0.05)$.

$\ddagger$ Mean values show minimum levels of circulating NEFA since they declined from initial levels after meal ingestion.

secretion ${ }^{(22)}$ increases fat oxidation and prevents high-fat dietinduced obesity in mice. In addition, higher blood GIP levels after GIP treatment significantly decreased fat utilisation in mice $^{(2)}$ and energy expenditure in human subjects ${ }^{(24)}$. The present results are consistent with the above studies, and suggest that increased fat oxidation after the HDP meal compared with that for the waxy maize starch meal might be driven by decreased circulating GIP after the ingestion of the former compared with the latter.

Lower postprandial glycaemic and insulinaemic responses after the HDP meal than those after the waxy maize starch meal were compatible with the results regarding other types
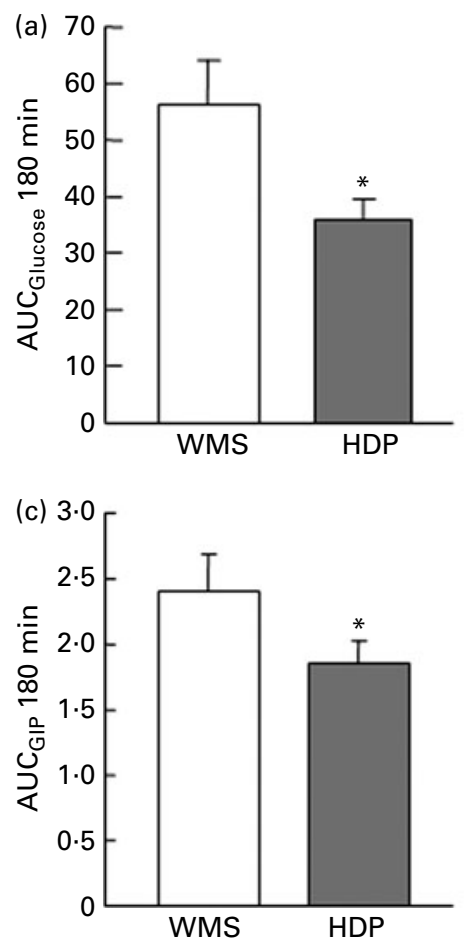

of RS (mostly RS2 type $)^{(11,12-15)}$. Raben et al. ${ }^{(14)}$ showed that postprandial glycaemia, insulinaemia and GIP responses are significantly lower after the intake of raw potato starch compared with the corresponding pregelatinised potato starch. In their study, RS (raw potato starch) and corresponding digestible starch (pregelatinised potato starch) were mixed into fruit syrup, which resulted in the test meals having a completely different texture, one a porridge-like gel and the other drinkable liquid ${ }^{(14)}$, with solid meals known to be associated with a delay in gastric emptying compared with liquid meals ${ }^{(27)}$. In contrast, the two pancake test meals in the present study have a similar texture, since pregelatinised HDP
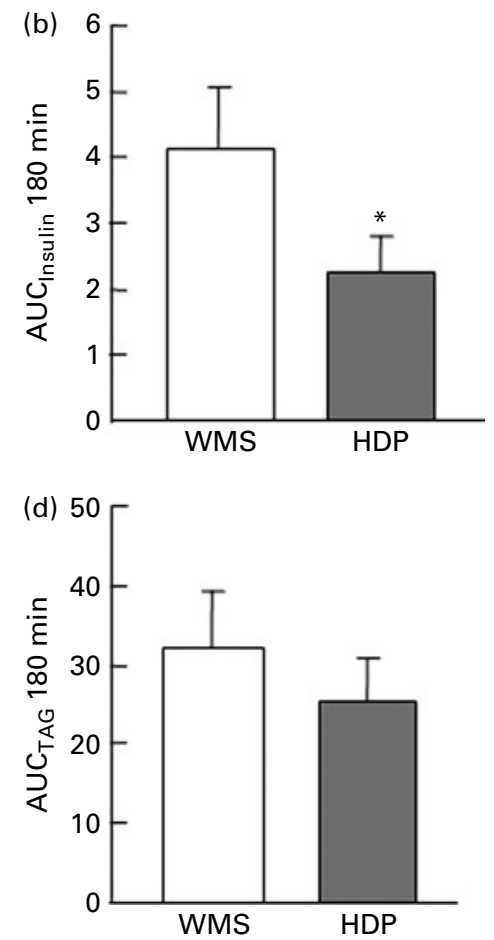

Fig. 4. Incremental area under the curve (AUC) for the (a) blood glucose, (b) insulin, (c) glucose-dependent insulinotropic polypeptide (GIP) and (d) TAG responses after the consumption of the test meal containing waxy maize starch (WMS) or hydroxypropyl-distarch phosphate (HDP; from waxy maize starch) in healthy male subjects. Values are means, with standard errors represented by vertical bars (ten subjects per group). Statistical analysis was conducted using the paired $t$ test. ${ }^{*}$ Mean values were significantly different $(P<0.05)$. 
Table 3. Effects of meals and time, and meal $\times$ time interactions for the area under the curve of metabolic variables ${ }^{\star}$

\begin{tabular}{lllll}
\hline Variables & Glucose & Insulin & GIP & TAG \\
\hline Meal & $<0.0001$ & $<0.01$ & $<0.01$ & NS \\
Time & $<0.0001$ & $<0.0001$ & $<0.0001$ & $<0.0001$ \\
Meal $\times$ time & NS $\dagger$ & NS $\dagger$ & NS $\dagger$ & NS $\dagger$ \\
interaction & & & & \\
\hline
\end{tabular}

GIP, glucose-dependent insulinotropic polypeptide.

* Statistical analysis was conducted using two-way repeated-measures ANOVA.

$\dagger$ Values were not significantly different $(P>0.05)$.

does not retrograde in the batter and remains highly gelatinised in the test meal as well as waxy maize starch. The peak time of either the blood glucose, insulin, GIP or TAG concentration was not delayed after HDP meal ingestion compared with that after the waxy maize starch meal. Therefore, the lower postprandial glycaemia, insulinaemia and GIP responses do not seem likely to be attributed to delayed gastric emptying after HDP meal consumption.

The extent of starch gelatinisation and fat content vary within many processed food products, being important factors in starch digestion and the postprandial metabolic response. In our preliminary experiments using mice, HDP stimulated lower blood glucose, insulin and GIP responses than waxy maize starch when ingested either with various amounts of fat (see Fig. S1 of the supplementary material, available online at http://www.journals.cambridge.org/bjn) or after being gelatinised to varying levels (see Fig. S2 of the supplementary material, available online at http://www.journals.

Table 4. Pearson's correlation coefficient between metabolic variables and energy utilisation†

\begin{tabular}{|c|c|c|c|}
\hline Variables & $\begin{array}{r}A_{\text {REE }} \\
180 \mathrm{~min}\end{array}$ & $\begin{array}{r}\mathrm{AUC}_{\mathrm{CHO}} \\
180 \mathrm{~min}\end{array}$ & $\begin{array}{l}\mathrm{AUC}_{\mathrm{FAT}} \\
180 \mathrm{~min}\end{array}$ \\
\hline$C_{0}$ (glucose) & -0.104 & 0.357 & -0.0274 \\
\hline$C_{0}$ (insulin) & -0.103 & -0.142 & 0.095 \\
\hline$C_{0}(\mathrm{GIP})$ & -0.080 & -0.080 & -0.035 \\
\hline$C_{0}$ (TAG) & -0.133 & -0.378 & 0.254 \\
\hline$C_{0}(\mathrm{NEFA})$ & -0.443 & -0.361 & 0.058 \\
\hline$C_{\max }($ glucose $)$ & -0.110 & 0.328 & -0.302 \\
\hline$C_{\max }($ insulin) & -0.145 & -0.217 & 0.149 \\
\hline$C_{\max }(\mathrm{GIP})$ & -0.363 & 0.081 & -0.358 \\
\hline$C_{\max }(\mathrm{TAG})$ & -0.306 & -0.403 & 0.175 \\
\hline$C_{\max }(\mathrm{NEFA})$ & $-0.529^{\star}$ & -0.286 & -0.010 \\
\hline$\Delta C_{\max }$ (glucose) & -0.044 & 0.103 & -0.130 \\
\hline$\Delta C_{\max }$ (insulin) & -0.145 & -0.219 & 0.150 \\
\hline$\Delta C_{\max }(\mathrm{GIP})$ & $-0.454^{\star}$ & 0.187 & $-0.486^{*}$ \\
\hline$\Delta C_{\max }(\mathrm{TAG})$ & $-0.584^{\star \star}$ & -0.270 & -0.105 \\
\hline$\Delta C_{\max }(\mathrm{NEFA})$ & 0.350 & 0.337 & -0.073 \\
\hline $\mathrm{AUC}_{\text {glucose }} 180 \mathrm{~min}$ & 0.028 & -0.184 & 0.139 \\
\hline $\mathrm{AUC}_{\text {insulin }} 180 \mathrm{~min}$ & -0.148 & -0.183 & $0 \cdot 105$ \\
\hline $\mathrm{AUC}_{\mathrm{GIP}} 180 \mathrm{~min}$ & $-0.576^{\star \star}$ & $0 \cdot 173$ & $-0.514^{*}$ \\
\hline $\mathrm{AUC}_{\mathrm{TAG}} 180 \mathrm{~min}$ & $-0.558^{\star}$ & -0.043 & -0.231 \\
\hline $\mathrm{AUC}_{\mathrm{NEFA}} 180 \mathrm{~min}$ & 0.359 & 0.293 & -0.016 \\
\hline
\end{tabular}

AUC $180 \mathrm{~min}$, area under the curve of metabolic variables for $180 \mathrm{~min}$ after test meal ingestion; REE, resting energy expenditure; $\mathrm{CHO}$, carbohydrate utilisation; FAT, fat utilisation; $C_{0}$, initial concentration of blood metabolic variables before and after test meal ingestion; $C_{\max }$, maximum concentration of blood metabolic variables before and after test meal ingestion; $\Delta C_{\max }$, maximum increase of blood metabolic variables from initial blood levels; GIP, glucose-dependent insulinotropic polypeptide.

${ }^{\star} P<0.05,{ }^{* *} P<0.01$.

† Pearson's correlation coefficients were obtained to estimate the linear correlation between two parameters. cambridge.org/bjn). The in vivo availability of highly gelatinised HDP as a carbohydrate (glucose) was equivalent to $70 \%$ of the unmodified starch in the corresponding starch:fat ratio $(1: 0 \cdot 2)$. The test meal in the present study contained $18.6 \%$ highly gelatinised starch (and $12.1 \%$ other carbohydrates) with a starch:fat ratio of 1:0.28. Therefore, carbohydrate dilution of the test meal with RS4 is estimated to be approximately $82 \%$. This carbohydrate dilution may explain the lower glycaemic and hormonal responses after the consumption of HDP than those for waxy maize starch. However, HDP supplementation of the meal decreased the postprandial glycaemia, insulinaemia and GIP responses to 63,55 and $77 \%$, respectively, compared with those for the waxy maize starch meal. The reason for these glycaemic and hormonal responses after HDP consumption being lower than expected based on the carbohydrate dilution needs to be elucidated.

Dietary carbohydrates and fats stimulate a postprandial increase in the blood GIP level ${ }^{(28-30)}$. GIP secretion from GIP-producing $\mathrm{K}$ cells is triggered by glucose and NEFA ${ }^{(31)}$. Although a lower GIP response after the ingestion of HDP could be explained partly by its decreased availability (glucose release by digestion) compared with that of waxy maize starch, we cannot rule out other possible factors that could have contributed to the postprandial glycaemic and GIP responses: (1) HDP ingestion alters gastric emptying, (2) HDP stimulates less GIP expression than waxy maize starch and (3) HDP lowers nutrient (GIP secretagogue) transport from the lumen to the intestinal epithelium, and thereby stimulates lower-level GIP secretion from $\mathrm{K}$ cells located in the intestinal epithelium. The hypothesis that HDP may modulate gastric emptying was not examined in the present study, since this was not observed in our previous study involving animals $^{(2)}$. Tseng et $a l .{ }^{(32)}$ showed that glucose ingestion increases duodenal mucosal GIP mRNA concentrations after $2 \mathrm{~h}$, and serum and mucosal GIP concentrations earlier, starting at $1 \mathrm{~h}$, indicating that duodenal GIP expression is stimulated at both pre- and post-translational levels by glucose-containing meals. Therefore, decreased GIP release after the HDP meal ingestion could be attributed to less mucosal GIP expression after the ingestion of HDP compared with that for waxy maize starch. In our preliminary experiments using everted jejunal sacs, pregelatinised HDP decreased the transport of both radiolabelled glucose and fatty acids from the lumen to the intestinal tissue compared with that of waxy maize starch (A Shimotoyodome, unpublished results). Gelatinised HDP has a marked water-holding capacity and viscosity, and is more stable in the presence of acids and digestive enzymes than the corresponding digestible starch. Thus, gelatinised HDP (and possibly its digested products also) might have inhibitory effects on the accessibility of dietary nutrients after digestion, on the rate of intestinal transport of nutrients and thereby on the secretion of gut hormones. However, the actual mechanisms behind decreased GIP secretion after HDP ingestion need to be investigated in future studies.

The present study is the first to show that the replacement of digestive starch with HDP (RS4-type RS) in a mixed meal can cause a significant increase in energy expenditure and fat oxidation in healthy individuals over the postprandial 

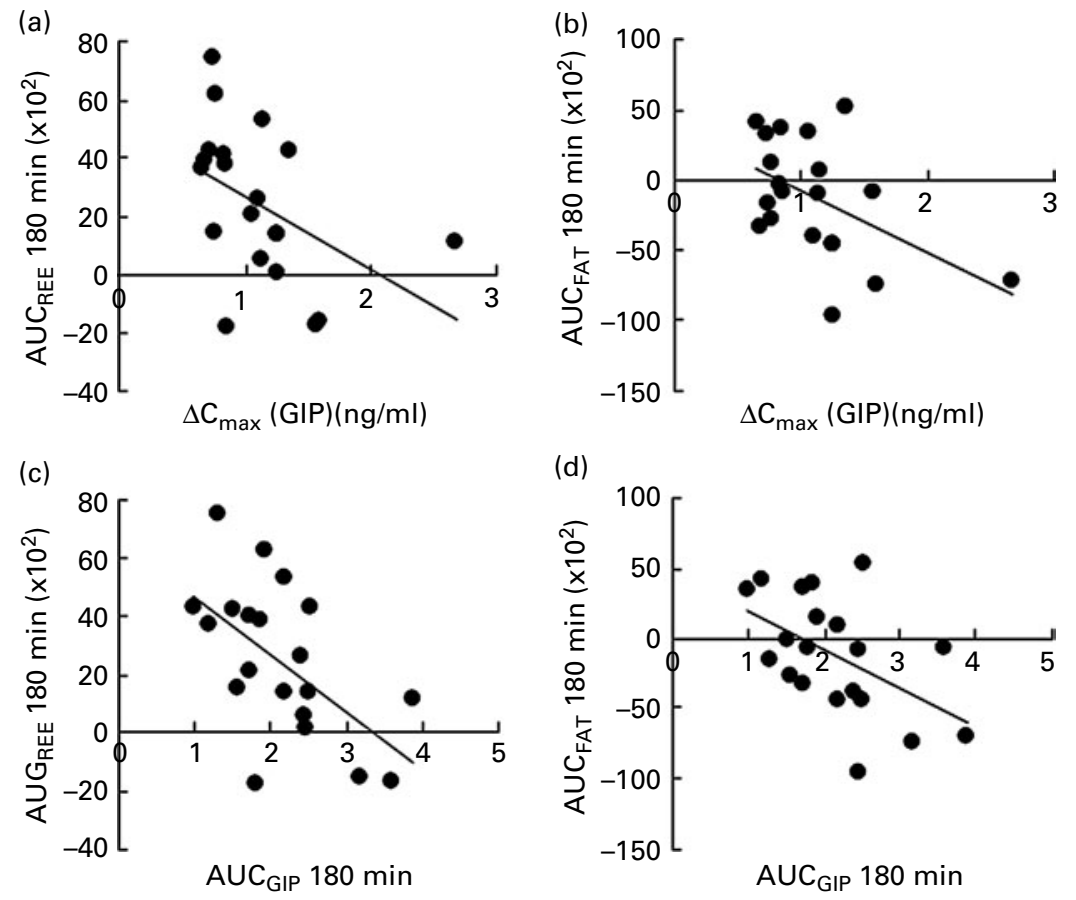

Fig. 5. Correlation between the postprandial blood glucose-dependent insulinotropic polypeptide (GIP) response and energy utilisation. Correlation of the maximum increase in blood GIP $\left(\Delta C_{\max }\right)$ after the meal, with area under the curve (AUC) for (a) resting energy expenditure (REE) $(R-0.454 ; P<0.05)$ and (b) fat utilisation (FAT) $(R-0.486 ; P<0.05)$. Correlation of the AUC for blood GIP $\left(\Delta C_{\max }\right)$ after the meal, with the AUC for (c) REE $(R-0.576 ; P<0.01)$ and (d) fat utilisation $(R-0.514 ; P<0.05)$. Pearson's correlation coefficients were obtained to estimate the linear correlation between two parameters. Correlations were considered significant when the error probability was smaller than 0.05 .

period. The increase in energy expenditure and fat oxidation was accompanied by a concomitant reduction in the postprandial GIP increase. These results may have beneficial implications for weight control, and help clarify the role of postprandial GIP release in the control of obesity. Indeed, the long-term ingestion of HDP, which reduces postprandial GIP release, attenuated high-fat diet-induced obesity in mice $^{(2)}$. Furthermore, the magnitude of the increase in energy expenditure and fat oxidation indicates that this effect is biologically relevant and could be important for preventing fat accumulation in the long term by influencing the total fat balance under chronic feeding conditions. However, further studies are required to confirm a similar efficacy in overweight or obese subjects, and to determine the exact mechanisms for the effect. Lower glycaemic and insulinaemic responses were also observed in the present study. Thus, daily HDP consumption in place of digestible starch might be beneficial to control postprandial hyperglycaemia and hyperinsulinaemia, and then improve impaired glucose tolerance and insulin resistance.

\section{Acknowledgements}

The present study was supported financially by Kao Corporation. The authors thank Yuki Ishida for his excellent technical support in manufacturing the pancake test meals used in the present study. The authors wish to thank all participants for their time and cooperation. The authors declare that there are no conflicts of interest to disclose. A. S. managed the human study, analysed the data and drafted the manuscript. J. S. was involved in the recruitment and management of the participants, and collection and analysis of the data. Y. K. designed and manufactured the test meals, and analysed their nutritional composition. T. H. was involved in the conception of the study, management of research expenses and the interpretation of data. All authors contributed to the designing of the study and the critical revision of the manuscript.

\section{References}

1. Englyst HN \& Cummings JH (1987) Resistant starch, a "new" food component: a classification of starch for nutritional purposes. In Cereals in a European Context, pp. 221-233 [ID Morton, editor]. Chichester: Ellis Horwood.

2. Shimotoyodome A, Suzuki J, Fukuoka D, et al. (2010) RS4type resistant starch prevents high-fat diet-induced obesity via increased hepatic fatty acid oxidation and decreased postprandial GIP in C57BL/6J mice. Am J Physiol Endocrinol Metab 298, E652-E662.

3. Björck I, Gunnarsson A \& Östergrd K (1989) A study of native and chemically modified potato starch. Part II: digestibility in the rat intestinal tract. Starch/Stärke 41, 128-134.

4. Ebihara K (1992) In vitro alpha-amylase hydrolysis of modified starch and postprandial plasma glucose response [in Japanese]. J Jpn Soc Nutr Food Sci 45, 551-553. 
5. Östergrd K, Björck I \& Gunnarsson A (1988) A study of native and chemically modified potato starch. Part I: analysis and enzymic availability in vitro. Starch/Stärke 40, 58-66.

6. Whistler RL \& Belfort AM (1961) Nutritional value of chemically modified corn starch. Science 133, 1599-1600.

7. Wootton M \& Chaudhry MA (1981) In vitro digestion of hydroxypropyl derivatives of wheat starch. I. Digestibility and action pattern using porcine pancreatic alpha-amylase. Starch/Stärke 33, 135-137.

8. Asp NG (1992) Resistant starch. Proceeding of the 2nd plenary meeting of EURESTA: European Flair Concerted Action No. 11 on physiological implication of the consumption of resistant starch in man [preface]. Eur J Clin Nutr 46, Supp. 2, S1.

9. Bird AR, Brown IL \& Topping DL (2000) Starches, resistant starches, the gut microflora and human health. Curr Issues Intest Microbiol 1, 25-37.

10. Annison G \& Topping DL (1994) Nutritional role of resistant starch: chemical structure vs physiological function. Annu Rev Nutr 14, 297-320.

11. Granfeldt Y, Drews A \& Bjorck I (1995) Arepas made from high amylase corn flour produce favorably low glucose and insulin responses in healthy humans. $J$ Nutr $\mathbf{1 2 5}$, 459-465.

12. Heijnen ML, van Amelsvoort JM \& Weststrate JA (1995) Interaction between physical structure and amylose:amylopectin ratio of foods on postprandial glucose and insulin responses in healthy subjects. Eur J Clin Nutr 49, 446-457.

13. Hoebler C (1999) Bioavailability of starch in bread rich in amylose: metabolic responses in healthy subjects and starch structure. Eur J Clin Nutr 53, 360-366.

14. Raben A, Tagliabue A, Christensen NJ, et al. (1994) Resistant starch: the effect on postprandial glycemia, hormonal response, and satiety. Am J Clin Nutr 60, 544-551.

15. Weststrate JA \& van Amelsvoort JM (1993) Effects of the amylose content of breakfast and lunch on postprandial variables in male volunteers. Am J Clin Nutr 58, 180-186.

16. Behall KM, Scholfield DJ \& Canary J (1988) Effect of starch structure on glucose and insulin responses in adults. $A m \mathrm{~J}$ Clin Nutr 47, 428-432.

17. Jenkins DJ, Vuksan V, Kendall CW, et al. (1998) Physiological effects of resistant starches on fecal bulk, short chain fatty acids, blood lipids and glycemic index. J Am Coll Nutr 17, 609-616

18. Ranganathan S, Champ M, Pechard C, et al. (1994) Comparative study of the acute effects of resistant starch and dietary fibers on metabolic indexes in men. Am J Clin Nutr 59, 879-883.

19. Tagliabue A, Raben A, Heijnen ML, et al. (1995) The effect of raw potato starch on energy expenditure and substrate oxidation. Am J Clin Nutr 61, 1070-1075.
20. Irwin N \& Flatt PR (2009) Therapeutic potential for GIP receptor agonists and antagonists. Best Pract Res Clin Endocrinol Metab 23, 499-512.

21. Song DH \& Wolfe MM (2007) Glucose-dependent insulinotropic polypeptide and its role in obesity. Curr Opin Endocrinol Diabetes Obes 14, 46-51.

22. Althage MC, Ford EL, Wang S, et al. (2008) Targeted ablation of glucose-dependent insulinotropic polypeptide-producing cells in transgenic mice reduces obesity and insulin resistance induced by a high fat diet. $J$ Biol Chem 283, 18365-18376.

23. Miyawaki K, Yamada Y, Ban N, et al. (2002) Inhibition of gastric inhibitory polypeptide signaling prevents obesity. Nat Med 8, 738-742.

24. Daousi C, Wilding JPH, Aditya S, et al. (2009) Effects of peripheral administration of synthetic human glucosedependent insulinotropic peptide (GIP) on energy expenditure and subjective appetite sensations in healthy normal weight subjects and obese patients with type 2 diabetes. Clin Endocrinol 71, 195-201.

25. Wahrlich V, Anjos LA, Going SB, et al. (2006) Validation of the VO2000 calorimeter for measuring resting metabolic rate. Clin Nutr 25, 687-692.

26. Weir J (1949) New methods for calculating metabolic rate with special reference to protein metabolism. J Physiol 109, 1-9.

27. Notivol R, Carrio I \& Cano L (1984) Gastric emptying of solid and liquid meals in healthy young subjects. Scand J Gastroenterol 19, 1107-1113.

28. Elliot RM, Morgan LM, Tredger JA, et al. (1993) Glucagonlike peptide-1 (7-36)amide and glucose-dependent insulinotropic polypeptide secretion in response to nutrient ingestion in man: acute post-prandial and 24-h secretion patterns. J Endocrinol 138, 159-166.

29. Meier JJ \& Nauck MA (2004) Glucose-dependent insulinotropic polypeptide/gastric inhibitory polypeptide. Best Pract Res Clin Endocrinol Metab 18, 587-606.

30. Shimotoyodome A, Fukuoka D, Suzuki J, et al. (2009) Coingestion of acylglycerols differentially affects glucoseinduced insulin secretion via glucose-dependent insulinotropic polypeptide in $\mathrm{C} 57 \mathrm{BL} / 6 \mathrm{~J}$ mice. Endocrinology 150, 2118-2126

31. Parker HE, Habib AM, Rogers GJ, et al. (2009) Nutrientdependent secretion of glucose-dependent insulinotropic polypeptide from primary murine K cells. Diabetologia $\mathbf{5 2}$, 289-298.

32. Tseng CC, Jarboe LA \& Wolfe MM (1994) Regulation of glucose-dependent insulinotropic peptide gene expression by a glucose meal. Am J Physiol 266, G887-G891.

33. Kainuma K, Matsunaga A, Itakawa M, et al. (1981) New enzyme system - $\beta$-amylase-pullulanase - to determine the degree of gelatinization and retrogradation of starch or starch products [in Japanese]. Denpun Kagaku 28, 235-240. 